\title{
A new Approach for Kalman filtering on Mobile Robots in the presence of uncertainties
}

\author{
Larsen, Thomas Dall; Andersen, Nils Axel; Ravn, Ole
}

Published in:

Proceedings of IEEE Conference on Control Applications

Link to article, DOI:

10.1109/CCA.1999.801002

Publication date:

1999

Document Version

Publisher's PDF, also known as Version of record

Link back to DTU Orbit

Citation $(A P A)$ :

Larsen, T. D., Andersen, N. A., \& Ravn, O. (1999). A new Approach for Kalman filtering on Mobile Robots in the presence of uncertainties. In Proceedings of IEEE Conference on Control Applications (Vol. 2). IEEE.

https://doi.org/10.1109/CCA.1999.801002

\section{General rights}

Copyright and moral rights for the publications made accessible in the public portal are retained by the authors and/or other copyright owners and it is a condition of accessing publications that users recognise and abide by the legal requirements associated with these rights.

- Users may download and print one copy of any publication from the public portal for the purpose of private study or research.

- You may not further distribute the material or use it for any profit-making activity or commercial gain

- You may freely distribute the URL identifying the publication in the public portal 


\title{
A New Approach for Kalman Filtering on Mobile Robots in the Presence of Uncertainties
}

\author{
Thomas Dall Larsen, Nils A. Andersen \& Ole Ravn \\ Department of Automation, Technical University of Denmark \\ Building 326, DK-2800 Lyngby, Denmark \\ E-mail: $\{t d l$, naa, or $\} @ i a u . d t u . d k \quad F a x: ~+4545881295$
}

Keywords: Mobile robot, modelling, Kalman filter, uncertainty

\begin{abstract}
In many practical Kalman filter applications, the quantity of most significance for the estimation error is the process noise matrix. When filters are stabilized or performance is sought improved, tuning of this matrix is the most common method. This tuning process cannot be done before the filter is implemented, as it is primarily made necessary by modelling errors. In this paper two different methods for modelling the process noise are described and evaluated; a traditional one based on Gaussian noise models and a new one based on propagating modelling uncertainties. It will be discussed which method to use and how to tune the filter to achieve the lowest estimation errors.
\end{abstract}

\section{Introduction}

The most common way in practice to prevent an erroneous filter model to bias or diverge the estimates, is to force the filter to put less confidence in the model and more in the measurements. This is done by increasing the filter's process noise covariance matrix, $Q$, which is equivalent to adding fictitious process noise in the model to simulate the uncertainties. As it is impossible to model a real robot perfectly, it is almost always necessary to tune $Q$ when Kalman filters are implemented. As this diminishes not only the influence of the modelling errors, but also of the model itself, some considerations should be made regarding the complexity of the model. A thorough and tedious attempt to model the robot followed by a tuning of $Q$ that in practice deteriorates or even discards the outputs from this model, is wasting time both in the design phase and during runtime.

Besides, trying to make an accurate dynamical model of the robot contemplating all the nonlinearities caused by for instance friction forces, is not a trivial task, and is hardly ever seen in the literature (one example though can be found in [1]). The problem (besides the nonlin$0-7803-5446-X / 99 \$ 10.00$ (c) 1999 IEEE earities) is that a lot of parameters that change with for instance time and temperature, are required to be known quite precisely. A model requiring only three physical parameters to be known precisely, can be obtained by using the odometric system of the robot as the system model as in [2] or [3]. Here readings from the robot encoders are used, not as measurements, but as inputs driving the filter model.

\section{Odometric Kalman Filter}

If the mobile robot is equipped with two driving wheels each mounted with an odometric sensor (encoder) a very feasible and common way of designing the posture estimator, is by using these encoder readings as the system model. In this approach, the encoder readings are translated to increases in the robot's translational and rotational position and used as inputs to a simple geometrical filter model. An example of such a robot with an additional passive wheel (a castor wheel) mounted in the back of the robot, is shown on figure 1. During

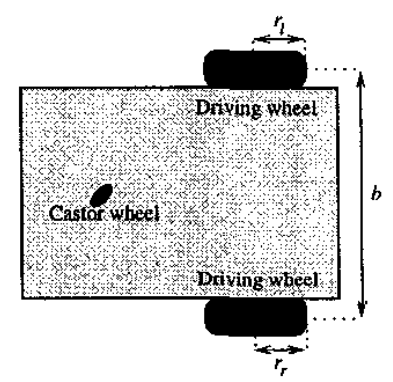

Figure 1: A mobile robot with a dual drive and encoder system.

one sample period, the encoders will measure angular increments corresponding to the distances $d_{r}$ and $d_{l}$, traveled by the right and the left wheel respectively. If the movement of the mobile robot is assumed circular 
$d_{r}$ and $d_{l}$ can be transformed to a translational and rotational displacement of the robot:

$$
\begin{aligned}
\delta d & =\frac{d_{r}+d_{l}}{2} \\
\delta \theta & =\frac{d_{r}-d_{l}}{b},
\end{aligned}
$$

where $b$ is the distance between the wheels.

The robot coordinates in a global coordinate frame can then be updated by (see [4]):

$$
\left[\begin{array}{c}
X_{k+1} \\
Y_{k+1} \\
\theta_{k+1}
\end{array}\right]=\left[\begin{array}{c}
X_{k} \\
Y_{k} \\
\theta_{k}
\end{array}\right]+\left[\begin{array}{c}
\delta d_{k} \cos \left(\theta_{k}+\frac{\delta \theta_{k}}{\delta \theta_{k}}\right) \\
\delta d_{k} \sin \left(\theta_{k}+\frac{\delta \theta_{k}}{2}\right) \\
\delta \theta_{k}
\end{array}\right]
$$

Equation (3) assumes linear motion within each sample period. Typically the sampling rate is so high compared to the angular velocity of the mobile robot that this approximation is very accurate.

The three coordinates $(X, Y, \theta)$ constitute the state vector, $x$, for the mobile robot, and are observed by some additional absolute measurements, $z$. These measurements are described by a nonlinear function, $c$, of the robot coordinates and an independent Gaussian noise process, $v$. Denoting the nonlinear function (3) $a$, and collecting $\delta d_{k}$ and $\delta \theta_{k}$ in an input vector $u_{k}=\left[\begin{array}{ll}\delta d_{k} & \delta \theta_{k}\end{array}\right]^{T}$ the mobile robot can be described by:

$$
\begin{array}{ll}
x_{k}=a\left(x_{k-1}, u_{k-1}, w_{k-1}\right), & w_{k} \sim N\left(0, q_{k}\right) \\
z_{k}=c\left(x_{k}, v_{k}\right), & v_{k} \sim N\left(0, r_{k}\right)
\end{array}
$$

A discrete extended Kalman filter can then be designed as in [3]:

$$
\begin{aligned}
\hat{x}_{k} & =a\left(\hat{x}_{k-1}, u_{k-1}\right) \\
P_{k} & =A_{k-1} P_{k-1}(+) A_{k-1}^{T}+Q_{k-1} \\
K_{k} & =P_{k} C_{k}^{T}\left[C_{k} P_{k} C_{k}^{T}+R_{k}\right]^{-1} \\
\hat{x}_{k}(+) & =\hat{x}_{k}+K_{k}\left[z_{k}-C_{k} \hat{x}_{k}\right] \\
P_{k}(+) & =\left[I-K_{k} C_{k}\right] P_{k},
\end{aligned}
$$

where:

$$
\begin{aligned}
A_{k} & =\left.\frac{\partial a}{\partial x_{k}}\right|_{x_{k}=\hat{x}_{k}(+), w_{k}=0} \\
& =\left[\begin{array}{ccc}
1 & 0 & -\delta d_{k} \sin \left(\theta_{k}+\frac{\delta \theta_{k}}{2}\right) \\
0 & 1 & \delta d_{k} \cos \left(\theta_{k}+\frac{\delta \theta_{k}^{2}}{2}\right) \\
0 & 0 & 1
\end{array}\right] \\
C_{k} & =\left.\frac{\partial c}{\partial x_{k}}\right|_{x_{k}=\hat{x}_{k}(+), v_{k}=0} \\
R_{k} & =\left.\frac{\partial c}{\partial v_{k}} r_{k} \frac{\partial c}{\partial v_{k}}\right|_{x_{k}=\hat{x}_{k}(+)}
\end{aligned}
$$

The only quantity remaining is the process noise matrix, $Q$. Two different methods for finding this are outlined in the next section.

\subsection{The Process Noise}

Though the odometry can be used to describe the motion of the mobile robot quite simple and accurately, the validity of the model is limited by a number of error sources contaminating the encoder outputs. These can be divided into two categories as in [5]:

- Systematic (continously present) errors:

- uncertain wheel diameters (diameters can bc unequal and their average can differ from nominal)

- misalignment of wheels

- uncertainty about the effective wheelbasi (due to nonpoint wheel contact with floor)

- Nonsystematic (event driven) errors:

- travel over uneven floors

- travel over unexpected objects on the floor

- wheel slippage (due to slippery floors, fas maneuvers, external and internal forces an nonpoint wheel contact)

Nonsystematic errors are unpleasant, as it is difficult t, predict an upper bound on these. Systematic errors on the other hand are of a more deterministic nature, bu do have the unfortunate quality of accumulating in tr. filter, leading the error on the estimate to grow withou bounds. In smooth indoor environments, systematic er rors usually constitute the main error source, provide. the robot is maneuvered gently. In more rough an. unstructured outdoor environments, nonsystematic $\mathrm{e}_{1}$ rors may dominate. As the focus here is on sanel controlled vehicles, driving on smooth and clear sus faces, nonsystematic errors will be ignored. The erro sources of concern are thus the inaccurately modellc wheelbase and wheel diameters.

2.1.1 Gaussian white noise processes: A the error sources all affect the outputs of the encode. or the interpretation of these, a common way of $d$ : scribing the noise, is as two stochastic processes cor taminating the encoder outputs. As the Kalman filt was derived with (and guarantees optimality for) a additive, zero mean Gaussian process noise, this seen. to be the obvious choice. Several authors (such as [4 therefore models the process noise by two independe. Gaussian noise processes added to the encoder outpit.

$$
\begin{array}{ll}
d_{r}^{*}=d_{r}+\varepsilon_{r}, & \varepsilon_{r} \sim N\left(0, \sigma_{d_{r}}^{2}\right) \\
d_{l}^{*}=d_{l}+\varepsilon_{l}, & \varepsilon_{l} \sim N\left(0, \sigma_{d_{l}}^{2}\right)
\end{array}
$$

What is read from the encoders is therefore, as ind cated by the asterisks, not the true distances travol: by the wheels. If the noise now is propagated to til 
input vector $u=[\delta d \delta \theta]^{T}$ of the Kalman filter one obtains:

$\delta d^{*}=\frac{d_{r}^{*}+d_{l}^{*}}{2}=\delta d+w_{d}, \quad w_{d} \sim N\left(0, \frac{\sigma_{d_{r}}^{2}+\sigma_{d_{l}}^{2}}{4}\right)$

$\delta \theta^{*}=\frac{d_{r}^{*}-d_{l}^{*}}{b}=\delta \theta+w_{\theta}, \quad w_{\theta} \sim N\left(0, \frac{\sigma_{d_{r}}^{2}+\sigma_{d_{l}}^{2}}{b^{2}}\right)$

The covariance of $\delta d^{*}$ and $\delta \theta^{*}$ is:

$$
\operatorname{cov}\left(\delta d^{*}, \delta \theta^{*}\right)=\frac{\sigma_{d_{r}}^{2}-\sigma_{d_{i}}^{2}}{2 b}
$$

It seems fair to assume that the variances of the noise on the two wheels are of the same magnitude, i.e. that $\sigma_{d}^{2}=\sigma_{d_{r}}^{2}=\sigma_{d_{l}}^{2}$. The covariance (4) then becomes zero and the following noise model is obtained:

$$
u^{*}=u+w_{u}, \quad w_{u} \sim N\left(0, Q_{m}\right),
$$

where:

$$
Q_{m, G}=\left[\begin{array}{cc}
\frac{\sigma_{d}^{2}}{2} & 0 \\
0 & \frac{2 \sigma_{d}^{2}}{b^{2}}
\end{array}\right]
$$

To obtain a noise vector $w \sim N(0, Q)$, contaminating the state vector $x$, the noise input matrix, $G$, is introduced:

$$
Q=G Q_{m, G} G^{T}
$$

Denoting $\phi_{k}=\theta_{k}+\frac{\delta \theta_{k}}{2}, G$ becomes:

$$
G=\left.\frac{\partial a}{\partial u}\right|_{x=\hat{x}}=\left[\begin{array}{cc}
\cos \phi & -\frac{1}{2} \delta d \sin \phi \\
\sin \phi & \frac{1}{2} \delta d \cos \phi \\
0 & 1
\end{array}\right]
$$

The only quantity that needs to be determined in this noise model is, $\sigma_{d}$. Often this is found by some trial and error method (terms such as observation, tuning and simulation are frequently encountered here).

\subsubsection{Propagating modelling uncertainty:} The Gaussian process noise was chosen more because of its mathematical convenience, than its ability to accurately describe the estimation errors. If it is assumed that the uncertainties on the physical parameters used in the robot model are of a size where they dominate over the stochastic effects, a different approach can be considered $^{1}$. Assume that the wheel radii, $r_{r}$ and $r_{l}$, and the wheelbase, $b$, are known only with some uncertainty (denoted by asterisks):

$$
r_{l}^{*}=r_{l}+\Delta r_{l}, \quad r_{r}^{*}=r_{r}+\Delta r_{r}, \quad b^{*}=b+\Delta b
$$

As a result, the filter input $u^{*}=\left[\delta d^{*} \delta \theta^{*}\right]^{T}$ will deviate from the "true" inputs:

$$
\delta d^{*}=\frac{\left(r_{r}+\Delta r_{r}\right) \alpha_{r}+\left(r_{l}+\Delta r_{l}\right) \alpha_{l}}{2}
$$

\footnotetext{
${ }^{1} \mathrm{~A}$ related approach can be seen in [6], where the process noise is modelled by three stochastic processes scaled by the sum of the absolute distances traveled by the wheels.
}

$$
\begin{aligned}
& =\delta d+\frac{\Delta r_{r} \alpha_{r}+\Delta r_{l} \alpha_{l}}{2} \\
\delta \theta^{*} & =\frac{\left(r_{r}+\Delta r_{r}\right) \alpha_{r}-\left(r_{l}+\Delta r_{l}\right) \alpha_{l}}{b+\Delta b} \\
& =\frac{b}{b+\Delta b}\left(\delta \theta+\frac{\Delta r_{r} \alpha_{r}-\Delta r_{l} \alpha_{l}}{b}\right)
\end{aligned}
$$

The worst case values are:

$$
\begin{aligned}
\delta d_{\text {max }}^{*} & =\delta d+\frac{\left|\Delta r_{r} \alpha_{r}\right|+\left|\Delta r_{l} \alpha_{l}\right|}{2} \\
\delta d_{\min }^{*} & =\delta d-\frac{\left|\Delta r_{r} \alpha_{r}\right|+\left|\Delta r_{l} \alpha_{l}\right|}{2} \\
\delta \theta_{\max }^{*} & =\frac{b\left(\delta \theta+\frac{\left|\Delta r_{r} \alpha_{r}\right|+\left|\Delta r_{l} \alpha_{l}\right|}{b}\right)}{b-\operatorname{sign}(\delta \theta)|\Delta b|} \\
\delta \theta_{\min }^{*} & =\frac{b\left(\delta \theta-\frac{\left|\Delta r_{r} \alpha_{r}\right|+\left|\Delta r_{l} \alpha_{l}\right|}{b}\right)}{b+\operatorname{sign}(\delta \theta)|\Delta b|}
\end{aligned}
$$

The uncertainties of the input vector can then be defined by:

$$
\begin{aligned}
\Delta \delta d & =\frac{\delta d_{\max }^{*}-\delta d_{\min }^{*}}{2}=\left|\Delta r_{r} \alpha_{r}\right|+\left|\Delta r_{l} \alpha_{l}\right| \\
\Delta \delta \theta & =\frac{\delta \theta_{\max }^{*}-\delta \theta_{\min }^{*}}{2} \\
& =\frac{b}{b^{2}-\Delta b^{2}}\left(|\Delta b \delta \theta|+\left|\Delta r_{r} \alpha_{r}\right|+\left|\Delta r_{l} \alpha_{l}\right|\right)
\end{aligned}
$$

As $b \gg \Delta b$ then $b^{2} \simeq b^{2}-\Delta b^{2}$ which makes the following approximation reasonable:

$$
\Delta \delta \theta=\frac{1}{b}\left(|\Delta b \delta \theta|+\left|\Delta r_{r} \alpha_{r}\right|+\left|\Delta r_{l} \alpha_{l}\right|\right)
$$

A matrix describing the squared uncertainties of $\delta d^{*}$ and $\delta \theta^{*}$ can then be defined by:

$$
Q_{m, u}=\left[\begin{array}{cc}
(\Delta \delta d)^{2} & 0 \\
0 & (\Delta \delta \theta)^{2}
\end{array}\right]
$$

This matrix can be used as the covariance matrix of the input vector to the Kalman filter. Once the input covariance matrix is attained, the Kalman filter process noise matrix, $Q$, can be calculated using:

$$
Q=G Q_{m, u} G^{T}
$$

Using equation (7) as the covariance matrix in the Kalman filter, has the very fortunate quality that the process noise will depend on the movement of the robot. If the robot does not move, the covariance of the state will not increase. In contrast, the covariance of the estimate using the process noise defined by the covariance in equation (5), will increase regardless of the robot motion ${ }^{2}$.

\footnotetext{
${ }^{2}$ Or rather the input noise matrix is unaffected by the robot motion; the process noise still depends on the motion through the noise input matrix, $G$.
} 


\section{Simulations}

Simulations are now performed, with the mobile robot moving down a corridor with guide marks placed on one of the walls, as shown in figure 2. Both $Q_{m, G}$

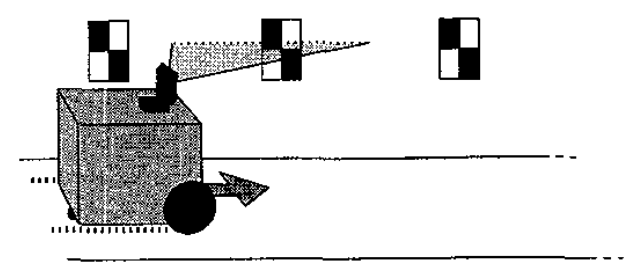

Figure 2: Mobile robot moving down corridor with guide marks on one wall.

and $Q_{m, u}$ are tried as input noise covariance matrices. When the matrix $Q_{m, u}$ is chosen, the uncertainties on the three physical parameters of the robot model need to be determined (the two wheel radii, $r_{r}$ and $r_{l}$, and the wheel base, $b$ ). These can be observed in a number of different ways, for instance by considering measurement accuracy, wheel deformation or drift. Here it will be assumed that they all lie within one per mille:

$\left|\Delta r_{r}\right|=0.001 r_{r}, \quad\left|\Delta r_{l}\right|=0.001 r_{l}, \quad|\Delta b|=0.001 b$

If $Q_{m, G}$ is chosen, a value for $\sigma_{d}^{2}$ will have to be found which is high enough to ensure that the effect of modelling errors are properly accounted for in the state covariance estimation. Else, the filter will be overconfident of its estimate and could diverge. Here, $\sigma_{d}$ is approximated to:

$$
\sigma_{d}=0.10 \cdot 10^{-3} \mathrm{~m}
$$

However, both $Q_{m, G}$ and $Q_{m, u}$ are scaled somewhat arbitrary; $Q_{m, G}$ because the variance of the assumed Gaussian noise process in (8) is unknown; $Q_{m, u}$ partly because it was created by "transforming" uncertainties into covariances, but also because the Kalman filter adds up squared uncertainties, when ideally it should add up the uncertainties themselves. These modelling difficulties make it likely that the estimation accuracy can be improved by scaling the covariance matrices by a scaling factor:

$$
Q_{m}=K_{\text {scale }} Q_{m, G}, \quad \vee \quad Q_{m}=K_{\text {scale }} Q_{m, u}
$$

The existence of an optimal scaling factor will now be proven in simulations and the optimal value found. The simulations are performed using an advanced nonlinear Simulink model (see [7]) of the mobile robot, contemplating both linear and nonlinear friction forces, as well as the dynamics of the mobile robot. The advantage of using simulations as opposed to physical experiments, is that the ground truth is known and the estimation errors therefore can be evaluated.

\subsection{The Scaling Factor}

The filter is run with the process noise matrix in (9) where first $Q_{m, G}$ and then $Q_{m, u}$ are tried. The modelling inaccuracies are chosen by a random generator as uniformly distributed numbers between plus/minus one per mille of the true values. For each value of $K_{\text {scale }}, 100$ simulations with different combinations of modelling inaccuracies are run. The results are shown in figure 3. From the figure it is seen that when the

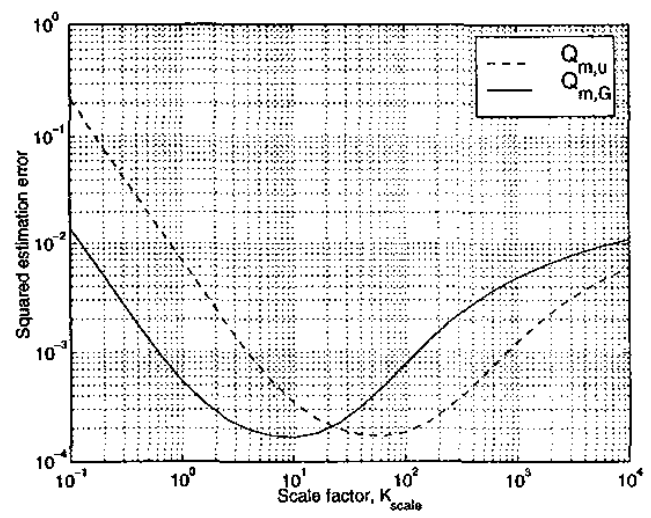

Figure 3: The sum of the squared estimation error, $\sum_{k} \tilde{x}_{k}^{T} \tilde{x}_{k}$, with different scaling factors, $K_{s c a l e}$.

mobile robot parameters are known with an accuracy of one per mille, the optimal value of the scaling factor in equation (9) is:

$$
K_{\text {scale }}=\left\{\begin{array}{ccc}
9 & \text { for } & Q_{m, G} \\
60 & \text { for } & Q_{m, u}
\end{array}\right.
$$

The two covariance matrices need different scaling, but show a similar dependence upon the scaling factor and yield the same estimation error when the respective optimal scaling factors are used.

The optimal value of $K_{\text {scale }}$ must be expected to change with the size of the uncertainties ${ }^{3}$. To examine this, the same experiment is now run with the maximum uncertainty varied between one and ten per milles. As before, each run is performed 100 times with the three model parameters, $r_{r}^{*}, r_{l}^{*}$ and $b^{*}$, chosen at random from a uniform distribution. The results can be seen in figure 4 . For the filters using the process noise matrix generated using modelling uncertainties $\left(Q_{m, u}\right)$, it is seen that the optimal scaling factor is almost constant for uncertainties above $\mathbf{0 . 3 \%}$. This suggests that if the uncertainties are in this range, the method describes the noise quite well. For very small modelling inaccuracies, other noise sources (friction, quantization, assumptions about lin-

\footnotetext{
${ }^{3}$ The optimal scaling factor must also be expected to change with the trajectory of the robot. However, this effect is not examined here.
} 


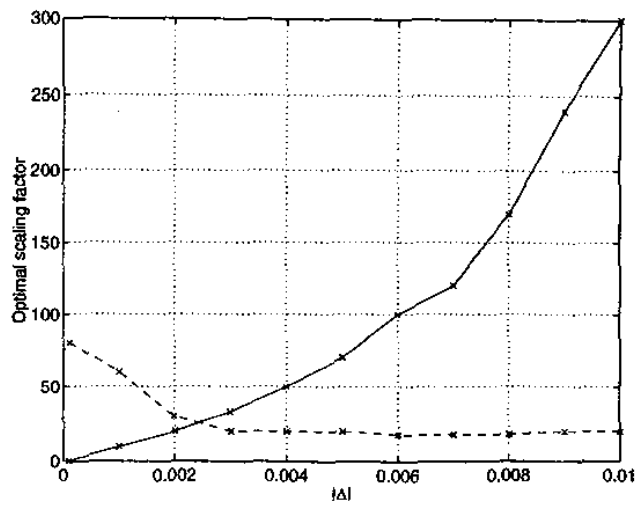

Figure 4: The optimal scaling factor, $K_{\text {scale }}$, with different maximum uncertainties and using the input noise matrices $Q_{m, G}$ and $Q_{m, u}$ (dashed).

ear movement, etc.) becomes dominant and the uncertainty description becomes inappropriate. Figure 4 shows that when the traditional Gaussian noise model is used, the optimal scaling factor increases exponentially with the size of the modelling errors. Hence, this method is much more sensitive to modelling errors. Given that the size of the modelling inaccuracies are estimated correctly, figure 4 can be used to determine whether to use the uncertainty description or the Gaussian model and in addition which scaling factor will yield the lowest estimation errors.

\subsection{The Estimation Error}

When the optimal scaling factors found above are used, the estimation errors for the two filters will propagate almost identically. If different scaling factors are used, the estimation error will increase more rapidly with the amplitude of the modelling inaccuracies. If a certain size of the inaccuracies are (wrongly) assumed and figure 3 is used to calculate the scaling factor, the estimation error will develope as shown in figure 5 . It is observed that the uncertainty based noise covariance matrix, $Q_{m, u}$, is more robust to modelling inaccuracies of unknown size and leads to lower estimation errors especially when the modelling errors are significantly higher than assumed.

\subsection{With big Uncertainties}

In figure 6 the optimal scaling factors for filters with quite big modelling uncertainties are shown. Observe that in the interval where the modelling errors are moderate (between $0.1 \%$ and $1 \%$ ), the scaling factor is constant and the uncertainty propagation method (the process noise matrix $Q_{m, u}$ ) is the most useful. When the uncertainties are lower, other errors are more significant. As the uncertainties grow the model becomes increasingly bad and useless. This means that when
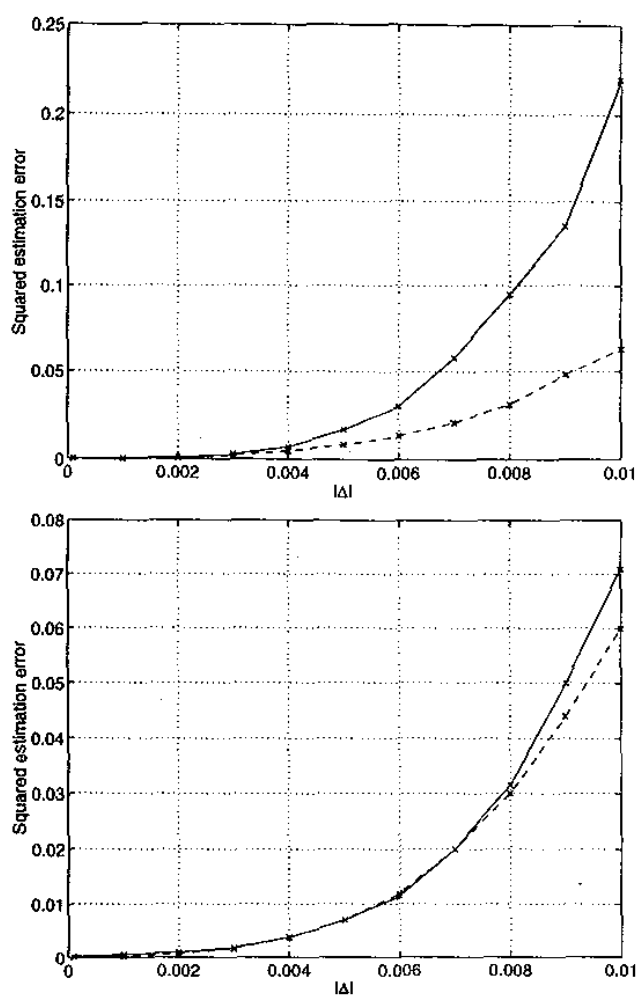

Figure 5: The sum of the squared estimation error with different maximum uncertainties for the input noise matrices $Q_{m, G}$ and $Q_{m, u}$ (dashed). The scaling factors, $K_{\text {scale }}$, are "wrongly" chosen assuming the uncertainties are $0.1 \%$ (upper plot) and $0.5 \%$ (lower plot).

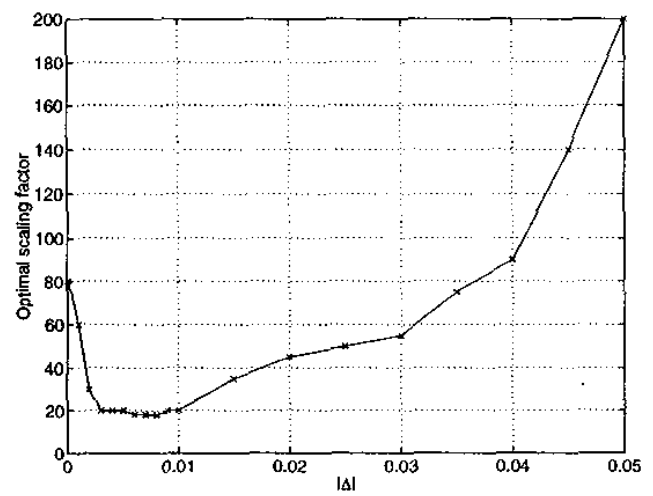

Figure 6: The optimal scaling factor, $K_{\text {scale }}$, for a large range of maximum uncertainties, using the input noise matrice $Q_{m, u}$.

the uncertainties are high, the exact value of the scaling factor is uncritical as long as it is high enough to ensure 
that the measurements are fused at a sufficiently high gain. This is illustrated in figure 7. It is seen that the
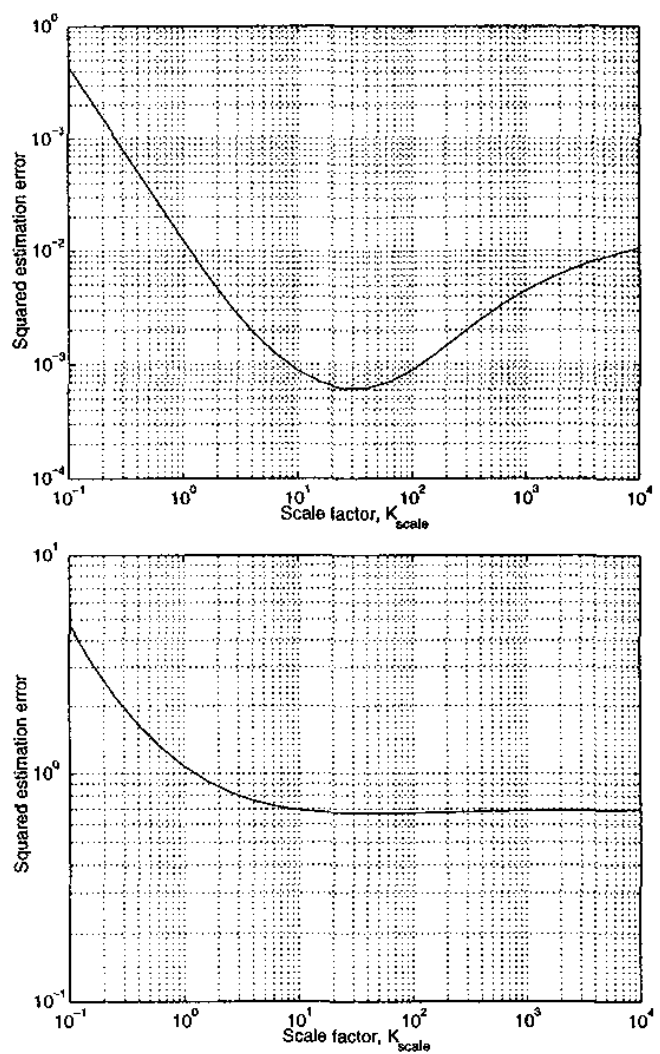

Figure 7: The sum of the squared estimation error using $Q_{m, u}$ with different scaling factors and maximum uncertainties of respectively $0.2 \%$ (upper plot) and $2 \%$ (lower plot).

higher the uncertainties are, the less it matters what the scaling factor is (as long as it is big). An optimal scaling factor still exists but if an arbitrary scaling factor higher than this is used, it will only affect the sum of the estimation error slightly.

\subsection{A More Intuitive Noise Propagation}

When the process noise is modelled by Gaussian sources with constant covariance, the covariance of the states are growing steadily, regardless of the speed of the robot. Clearly, it is not very intuitive that the estimation errors should grow when the robot posture (as well as the estimate of this) is constant. This means that the measurements, when the robot is moving slowly or the first measurement after the robot has been inanimate, will be fused at too high a gain. If instead uncertainty propagation is used the process noise will depend more closely on the trajectory of the robot and the covariance of the states will therefore grow slower at slower speeds and freeze when the robot is inanimate.

\section{Conclusion}

Simulations were made with two different types of process noise matrices - a traditional one using Gaussian white noise processes and a new one based on propagating modelling uncertainties. It was found that both of the process noise matrices needed scaling to minimize the estimation error. The new method was shown to be more robust in the presence of modelling uncertainties. The scaling factor for the traditional Gaussian noise model was shown to vary heavily with the size of the modelling uncertainties, but shown to be rather constant for the new method when the modelling uncertainties are large enough to be significant and yet so small that the model still is useful. This means that when the size of the uncertainties not are known precisely, the new covariance matrix will still be scaled close-to-optimal and therefore lead to significantly lower estimation errors.

\section{References}

[1] Gert L. Andersen, Anders C. Christensen, and Ole Ravn. Augmented models for improving vision control of a mobile robot. In $3 r d$ IEEE Conference on Control Applications, 1994.

[2] Satoshi Murata and Takeshi Hirose. Onboard locating system using real-time image processing for a self-navigating vehicle. IEEE Transactions on Industrial Electronics, 40(1), February 1993.

[3] F. Chenavier and J. L. Crowley. Position estimation for a mobile robot using vision and odometry. In Proceedings of the 1992 IEEE International Conference on Robotics And Automation, Nice, France, 1992.

[4] C. M. Wang. Location estimation and uncertainty analysis for mobile robots. In Proceedings of the 1988 International Conference on Robotics and $A u$ tomation, 1988.

[5] J. Borenstein and L. Feng. Measurement and correction of systematic odometry errors in mobil robots. IEEE Transactions on Robotics and Automa tion, 12(6), December 1996.

[6] E. Freund and F. Dierks. Map-based free navi gation for autonomous vehicles. Int. jounal of System. Science, 27(8), 1996.

[7] Magnus Nørgaard, Niels K. Poulsen, and O1: Ravn. The AGV-sim ver. 1.0 - a simulator for at: tonomous guided vehicles. Technical report, Depart ment of Automation, IAU, 1998. 